\title{
Boas práticas de cuidado às pessoas que usam drogas no contexto da atenção psicossocial
}

\author{
Good care practices for people who use drugs in the context of psychosocial care
}

Buenas prácticas de atención a las personas que consumen drogas en el contexto de la atención psicosocial

\section{Resumo}

Objetivo: refletir sobre as boas práticas em saúde mental no cuidado às pessoas usam drogas no contexto de atenção psicossocial. Método: estudo teórico reflexivo sustentado em revisão narrativa de literatura, nas bases de dados Literatura Latino-Americana e do Caribe em Ciências da Saúde e PubMed/Medical Literature Analysis and Retrieval Sistem on-line, sendo os critérios de seleção: artigos em português, inglês e espanhol; disponíveis na íntegra, que retratassem a temática referente à reflexão das boas práticas, nos últimos vinte anos. Resultados e discussão: para discutir e refletir sobre as boas práticas em saúde mental, foram criadas quatro categorias temáticas: Acesso aos serviços e a utilização da Rede de Atenção à Saúde (RAS) pelos usuários de Substâncias Psicoativas; Protagonismo e autonomia da pessoa no cuidado em saúde mental; O cuidado humanizado no território e a Redução de Danos; e Incorporação da família no cuidado. Conclusão: apesar dos avanços identificados na perspectiva de cuidado psicossocial, ainda é necessário demonstrar a importância dos serviços comunitários que atendem pessoas que usam drogas.

Palavras-chave: Saúde mental; Redução do dano; Usuário de droga; Prática clínica baseada em evidências.

\begin{abstract}
Objective: reflect on good mental health practices in caring for people who use drugs in the context of psychosocial care. Method: reflective theoretical study supported by a narrative review of literature, in the Latin American and Caribbean Literature in Health Sciences and PubMed/Medical Literature Analysis and Retrieval online databases, with the selection criteria: articles in Portuguese, English and Spanish; available in full, which portrayed the theme related to the reflection of good practices in the last twenty years. Results and discussion: to discuss and reflect on good practices in mental health, four thematic categories were created: Access to services and use of the Health Care Network (RAS) by users of Psychoactive Substances; Protagonism and autonomy of the person in mental health care; Humanized care in the territory and harm reduction; and Incorporation of the family into care. Conclusion: despite the advances identified in the perspective of psychosocial care, it is still necessary to demonstrate the importance of community services that serve people who use drugs.
\end{abstract}


Keywords: Mental health; Harm reduction; Drug user; Evidence-based clinical practice.

\section{Resumen}

Objetivo: reflexionar sobre las buenas prácticas de salud mental en el cuidado de personas que consumen drogas en el contexto de la atención psicosocial. Método: estudo teórico reflexivo sustentado em revisão narrativa de literatura, nas bases de dados Literatura Latino-Americana e do Caribe em Ciências da Saúde e PubMed/Medical Literature Analysis and Retrieval Sistem on-line, sendo os critérios de seleção: artigos em português, inglés y español; disponible en su totalidad, que retrató el tema relacionado con la reflexión de buenas prácticas en los últimos veinte años. Resultados y discusión: para discutir y reflexionar sobre buenas prácticas en salud mental, se crearon cuatro categorías temáticas: Acceso a servicios y uso de la Red de Salud (RAS) por usuarios de Sustancias Psicoactivas; Protagonismo y autonomía de la persona en la atención a la salud mental; Atención humanizada en el territorio y reducción de daños; e Incorporación de la familia al cuidado. Conclusión: a pesar de los avances identificados en la perspectiva de la atención psicosocial, aún es necesario demostrar la importancia de los servicios comunitarios que atienden a las personas que consumen drogas.

Palabras clave: Salud mental; Reducción de daños; Usuario de drogas; Práctica clínica basada en la evidencia.

\section{Introdução}

A Reforma Psiquiátrica propõe um modelo assistencial substitutivo às internações em manicômios, passando de um enfoque exclusivamente organicista e biológico, para uma abordagem multicausal do sofrimento psíquico, em que se evidencia a rede de saúde mental e os serviços comunitários como forma de cuidado psicossocial (Brasil, 2004). Neste sentido, pensa-se que para avaliar melhorias na atenção em saúde mental na perspectiva psicossocial, deve-se priorizar serviços que embasam as boas práticas baseadas em evidências cientificas (Thornicroft \& Tonsella, 2010).

No que concerne o cuidado às pessoas que sofrem em decorrência do uso abusivo de drogas, na compreensão biologicista, as evidências estão fortalecidas na neurociência - a partir de estudos que indicam efeitos produzidos pelas substâncias psicoativas e as ligações da dependência química no sistema neurológico. Na percepção social e cultural, as pesquisas desenvolvidas, levam em consideração as abordagens cognitivas comportamentais (Botega, 2012). Todavia, o cuidado no entendimento da atenção psicossocial, examina as relações subjetivas das pessoas em sofrimento psíquico e pode ser compreendido sobre a perspectiva de experiência da clínica e do relato de caso, novas ferramentas da saúde mental que podem auxiliar profissionais que trabalham com esta área (Antonaci, 2016).

O modelo neurobiológico da "dependência química" é aquele no qual predominam estudos clínicos e não consideram outras variáveis que influenciam o uso abusivo de drogas, como: sofrimento humano, relações conflitantes do sujeito, falta de perspectiva de vida, questões sociais, etc. Em 1978, o pesquisador Bruce Alexander, questionando essa abordagem limitada de pensar o cuidado em saúde mental, revelou falhas metodológicas neste tipo de investigação científica, considerando que estas pesquisas obtinham caminhos científicos estritamente farmacológicos e comportamentais para explicar um fenômeno tão complexo como é o mundo das drogas. O autor revelou cientificamente, realizando o "parque dos ratos", que usuários de drogas não usavam Substâncias Psicoativas (SPA) por uma necessidade essencialmente química, mas por não terem outras opções além da droga (Alexander, 1978).

As pesquisas com ratos e que embasavam o cuidado humano exclusivamente com abordagem em abstinência, ofereciam apenas duas opções de consumo para os animais de laboratório: água e água com cocaína. Alexander intrigado com as metodologias destes estudos, resolveu ampliar as possibilidades para os roedores, realizando um parque de diversões, em que estes obtinham outras opções de entretenimento, além da cocaína. Com o experimento, foi possível identificar que o consumo da droga diminui significativamente e até mesmo foi cessado por alguns animais. Alexander assim concluiu que a falta de opção para emoções prazerosas é o que pode desencadear o uso abusivo ou vício químico, popularmente conhecido.

O "vício", na perspectiva de Alexander, foi caracterizado enquanto uso rotineiro de determinada SPA e associado com situações em que a pessoa vive, não mais entendido a partir das propriedades químicas da droga em si. As circunstâncias que não oferecem redenção imediata, podem ser substituídas pelo uso de determinada droga - que modificam o estado de 
consciência momentaneamente e contribuem no desvio do foco causador do sofrimento humano. Neste sentido, o "vício", a partir dos experimentos de Alexander com o parque dos ratos, foi desmistificado e identificado como uma estratégia, um estilo de vida ou forma de encarar determinada situação difícil. No entanto, quando se obtém outras formas de enfrentar as circunstâncias da vida, sejam: relações sociais, diversão, dentre outras, é possível reverte-lo (Alexander, 1978).

Neurocientista norte americano, Carl Hart, ao realizar um estudo com pessoas usuárias de drogas, revelou que o poder da droga é inexistente, sendo o ambiente - principal fator desencadeador do uso abusivo. Esse pesquisador, proporcionou refletir sobre a desmistificação das terapêuticas autoritárias, que não condizem com as limitações impostas pela pessoa em sofrimento. Hart (2013), a partir desta produção científica, chamou atenção para a importância de se olhar sensivelmente o contexto do ser humano que muitas vezes, pela dificuldade de encará-lo, pode iniciar o uso desenfreado de drogas.

Nessa perspectiva, novos rumos da ciência possibilitaram propor cuidados que são compatíveis às restrições singulares. Os serviços de saúde podem ofertar possibilidades de cuidado adaptados as questões frágeis da pessoa em que, para aqueles que buscam sessar o uso abruptamente, a abstinência - e para os que não conseguem, outras alternativas, e/ou, Redução de Danos.

A redução de danos pode ser compreendida enquanto desenvolvimento de boas práticas nos serviços que atendem pessoas que usam drogas. Esta modalidade de cuidado teve início na Inglaterra, em 1926; na Holanda, em 1984. No Brasil, a primeira experiência ocorreu em 1989 - em Santos, com a distribuição de seringas e agulhas estéreis para usuários de heroína injetável, como forma de prevenir a propagação do HIV e Hepatites Virais. Foi expandida posteriormente para âmbito do uso de todas as drogas, legais e ilegais (Camargo et al., 2019). Atualmente a RD compõe a Política Nacional de álcool e outras drogas do Ministério da saúde e ampliou suas ações para a esfera do direito à saúde, cidadania e Direitos Humanos. Ela busca socialização política de usuários de drogas de maneira crítica, com protagonismo político, autonomia, promoção e prevenção de saúde na esfera individual e coletiva (Silva et al., 2018).

Desta forma, o desafio para realização do cuidado a essas pessoas com eficiência e sensibilidade, encontra alicerce nas boas práticas que sustentem o modelo psicossocial. Sendo as boas práticas, responsáveis por produzirem resultados efetivos na vida das pessoas que usam drogas abusivamente. Neste sentido, este artigo tem por objetivo, refletir sobre as boas práticas de cuidado às pessoas que usam drogas no contexto da atenção psicossocial.

\section{Metodologia}

Este artigo trata-se de um estudo teórico reflexivo sustentado em revisão narrativa de literatura nas bases de dados: Literatura Latino-Americana e do Caribe em Ciências da Saúde (LILACS) e PunMed/Medical Literature Analysis and Retrieval Sistem on-line (Medline). Foram utilizados para busca dos artigos os seguintes descritores em saúde (DECS) e suas combinações nas línguas portuguesa, inglesa e espanhola: Serviços de Saúde Mental (AND) usuários de drogas, e assim buscando responder à questão norteadora: o que tem sido publicado na literatura científica sobre as boas práticas em saúde mental, no cuidado às pessoas que usam drogas no contexto de atenção psicossocial?

Os critérios de inclusão definidos para a seleção dos artigos, foram: artigos publicados em português, inglês e espanhol; artigos na íntegra que retratassem a temática referente à reflexão das boas práticas, publicados e indexados nos referidos bancos de dados nos últimos vinte anos. Optou-se pela ampliação do período de publicação dos estudos, devido à dificuldade de se encontrar discussões sobre essa temática nos referidos bancos de dados. Foram excluídos da revisão narrativa, artigos que tratavam de transtornos severos e persistentes, saúde mental infantil e população de rua, devido a especificidade e afastamento do objetivo deste artigo.

Os estudos encontrados nesta busca que suscitaram a reflexão, versavam sobre os aspectos do processo de trabalho com boas práticas em saúde nos serviços de saúde da Rede de Atenção Psicossocial (RAS), Rede de Atenção Psicossocial 
(RAPS) que atendem pessoas que sofrem em decorrência do uso abusivo de Substâncias Psicoativas, dispositivos da Rede Informal que contribuem em novas abordagens em saúde mental e Redução de Danos. Dos 10 artigos encontrados, 9 obtinha metodologicamente abordagem qualitativa.

Apesar da revisão narrativa não se atentar rigorosamente para os passos metodológicos, contribui na discussão sobre determinado tema e suscita a reflexão a partir da compreensão de diferentes ambientes e suas relações, que são transversais a temática estudada (ZILMER, DÍAZ-MEDINA, 2018). Os artigos utilizados e que contribuíram para esta reflexão, foram selecionados no mês de maio de 2020 .

\section{Resultados e Discussão}

Considerando a proposta deste estudo, as reflexões sobre as boas práticas de cuidado às pessoas que usam drogas no contexto da atenção psicossocial, autores e leituras realizadas com a revisão narrativa, foram julgados cruciais a serem abordados neste artigo, a partir de quatro categorias, sendo elas: Acesso aos serviços e a utilização da Rede de Atenção à Saúde (RAS) pelos usuários de Substâncias Psicoativas; Protagonismo e autonomia da pessoa no cuidado em saúde mental; O cuidado humanizado no território e a Redução de Danos; e Incorporação da família no cuidado.

\section{Acesso aos serviços e a utilização da Rede de Atenção à Saúde (RAS) pelos usuários de Substâncias Psicoativas}

O acesso, acolhimento, as relações respeitosas entre os usuários e profissionais, são cruciais para obtenção de resultados terapêuticos positivos. $\mathrm{O}$ acolhimento, enquanto escuta, receptividade para com a pessoa que chega ao serviço de saúde mental (Pinho et al., 2017). torna-se a primeira ação terapêutica, importante para a criação do vínculo com o usuário. Esta estratégia transforma as relações entre profissionais e usuários, reverencia confiança e transparência no cuidado. Uma vez vinculado ao serviço, o sujeito consegue expor suas ideias e angústias no tratamento em saúde mental.

Em um CAPS de Minas Gerais, os aspectos positivos apontados pelos usuários, estavam atrelados ao acolhimento e o amparo para o deslocamento até o serviço, crucial para manutenção do processo terapêutico. O vínculo, a disposição dos profissionais em tempo integral do turno de trabalho, foi apontado como forte estratégia, que possibilitou o diálogo e a superação das inseguranças. Essas dinâmicas facilitaram a obtenção de informações no momento em que surgem as dúvidas. As oficinas também foram mencionadas como ações desenvolvidas pelo Centro de Atenção Psicossocial (CAPS), satisfatórias pelos participantes da pesquisa (Silva, Lima \& Ruas, 2018).

Pode-se se considerar também enquanto boas práticas no cuidado, o acesso e a itinerância dos usuários de SPA aos diferentes dispositivos das redes de atenção à saúde (RAS). Estudo realizado com enfermeiros de Estratégia e Saúde da Família (ESF), foi possível levantar que os profissionais acolhem o público de usuários de SPA. A maioria, assume essa responsabilidade e também entende que a Unidade Básica de Saúde (UBS), faz parte da Rede de Atenção à saúde mental (RAPS) (Silva et al., 2016).

\section{Protagonismo e autonomia da pessoa no cuidado em saúde mental}

Considerando a complexidade imersa no campo do uso abusivo de drogas e a multiplicidade dos fatores relacionados, sabe-se que, além de facilitarem o acesso aos serviços de saúde mental, os trabalhadores, devem obter a capacidade de vislumbrar as subjetividades do ser humano (Tavares et al., 2021).

Para que o usuário consiga superar as dificuldades relacionadas ao uso abusivo, o profissional deve adquirir a habilidade de elencar aspectos invisíveis do sujeito em sofrimento, compreendê-los, para motivar sua mudança de comportamento. O profissional é o apoiador, motivador do usuário em seu processo terapêutico, ele busca fortalecer os fatores de proteção, minimizando e se possível afastando, os fatores de riscos à saúde interligados ao uso abusivo de drogas. Assim, 
compreende-se a importância do protagonismo do usuário no seu processo de reabilitação psicossocial, visto que, é a partir da motivação e acreditando nas potencialidades que são singulares, que se é possível enfrentar e superar os malefícios do uso abusivo (Franchini, Rodrigues \& Tavares, 2017).

Essa perspectiva de cuidado, foi proporcionada com o rompimento cultural biomédico, na perspectiva de um tratamento que era centrado nos manicômios, com a exclusão do indivíduo socialmente e o aprisionamento em hospitais psiquiátricos. Esse modelo, além de não reinserir o indivíduo, deixava marcas indeléveis, como o rótulo de "louco", que impossibilitava a reintegração social e dificultava o desenvolvimento e a produção de vida dessas pessoas na sociedade.

O novo modelo de assistência em saúde mental, pauta-se em uma perspectiva contra hegemônica e antimacomial, evidenciando e assegurando o protagonismo do usuário no seu processo terapêutico, sendo este processo:-em sociedade, com relações afetivas e no território. O cuidado em liberdade possibilitou traçar estratégias, em que, considera-se o sujeito em sofrimento enquanto principal elemento do processo terapêutico. É a partir dele que a reabilitação será possível.

O território enquanto espaço de vida das pessoas que usam abusivamente alguma SPA, ao mesmo tempo que desencadeia as fragilidades para o uso, proporciona transformações e reabilitação. O CAPS, neste sentido, é o dispositivo institucional que facilita este processo no contexto particular de cada pessoa em sofrimento em decorrência do uso e abuso. Este serviço deve considerar a importância da participação e responsabilização do usuário, na perspectiva de Redução de Danos, para o que assim ocorra o autocuidado. A literatura revela que boa parte dos usuários que chegam ao serviço de saúde mental, buscam a abstinência como direcionamento de cuidado, todavia, na maioria das vezes, por não conseguirem pausar o uso abruptamente, recorrem a manutenção e controle do uso supervisionado pelos trabalhadores do serviço (Redução de Danos) (Silva, Lima \& Ruas, 2016).

Neste sentido, o Projeto Terapêutico Singular (PTS) é a ferramenta intermediadora do diálogo entre todos envolvidos no processo, para traçar a linha de cuidado. Apesar das evidências científicas mencionarem a importância desta ferramenta, o PTS, ainda é passível de grandes dificuldades em sua elaboração: alguns profissionais não tem clareza de como deve ser utilizado, não conseguem descrever metodologicamente o plano alinhavado com o usuário, ou ainda, propõem objetivos dissonantes com aqueles propostos pelo usuário, o que torna essa ferramenta importante de cuidado, em uma proposição unilateral (Silva, Abbad \& Montezano, 2018).

A construção do PTS proporciona pensar, repensar o cuidado, vislumbrando os elementos desencadeadores do uso abusivo de drogas. Se utilizado corretamente, poderá facilitar o processo terapêutico. O PTS considera os anseios e as possibilidades terapêuticas do usuário, traceja possibilidades e pode ser significativo nas mudanças de atitude. Salienta-se que este, não é um dispositivo inerte, tendo a ser seguido rigorosamente. Ele tem de ser flexível e considerar a clínica ampliada e as características particulares de cada usuário, projetos de vida - e ser reelaborado, quantas vezes forem necessárias (Silva, Abbad \& Montezano, 2018).

\section{O cuidado humanizado no território e a Redução de Danos}

A utilização do PTS, deve considerar o território e seus elementos nele contidos (igreja, UBS, escola, associações de moradores, vizinhos, família, etc). O território é um potente dispositivo a ser acionado no cuidado as pessoas que usam abusivamente substâncias psicoativas, sejam elas, legais ou ilegais. O profissional deve incentivar o usuário a utilizá-lo. Todavia, há dificuldades de controle sob as relações do usuário neste contexto, de se estar consciente das interações em seus diferentes cenários. Entretanto, a partir do vínculo é possível ambientar-se da vivência local e conduzir a terapêutica. $\mathrm{Na}$ prática do processo de trabalho do CAPS, os trabalhadores de saúde, compreendendo o espaço do usuário singularmente e levantando os dispositivos disponíveis a partir da escuta qualificada, entendendo a dinamicidade deste lugar, poderão ofertar 
diversificadas opções para o cuidado, sem impor metas do trabalhador ao usuário (Silva, Lima \& Ruas, 2018; Silva, Abbad \& Montezano, 2018).

O território, pode ser considerado um ótimo dispositivo a ser acionado no cuidado em saúde mental. Os profissionais do CAPS, são capazes de percorrer este espaço e identificar novas possibilidades extra muros da instituição terapêutica. Conhecer o território, proporciona conhecer a vida íntima das pessoas, facilitando o desenvolvimento de projetos de vida e a interlocução com seus elementos. Sendo a visita domiciliar, ação importante do serviço de saúde, ferramenta para que a equipe possa ambientar-se, conhecer a performance da família como um todo, compreendendo as inquietações referentes a moradia, bem como, os aspectos socioeconômicos que impactam a vida do usuário. Para mais, essa estratégia é fundamental para acolher a família, fortalecer os vínculos, dirimir geradores de estresse - muitas vezes motivadores do uso abusivo de drogas. Quando se conhece o território, a dinâmica familiar, as relações proximais e distantes do usuário devido algum desafeto, consegue-se ter dimensão do lugar que a substância ocupa na vida da pessoa e reduzir os danos advindos desse uso. Isso facilita a construção dos projetos terapêuticos, reduz danos advindos do uso de drogas, perpassando a ideia do cuidado centralizado na doença. Olhar para o território como possibilidade de reabilitação, requer criatividade e intersecção de múltiplos conhecimentos envolvidos, para que assim, consiga-se vislumbrar as múltiplas necessidades interligadas ao indivíduo em sofrimento (Silveira et al., 2016).

No estudo realizado em um serviço de território foi possível entender a importância da Redução de Danos como política de cuidado em saúde mental, que respeita e considera os limites das pessoas que usam a SPA, sendo eficiente para atenuação do consumo e riscos do uso inadequado de drogas. A Redução de Danos demonstra-se eficiente por ser desenvolvida no contexto, local do cotidiano do indivíduo: já que é nesse espaço em que estão imersos os disparadores do uso e singularidades, intimidades da pessoa (Oliveira et al., 2016).

\section{Incorporação da família no cuidado}

Em estudo realizado em um Centro de Atenção Psicossocial - Álcool e outras Drogas (CAPS-AD) (SILVEIRA, et al, 2016), foi possível compreender a importância da família no processo terapêutico, na atenuação de internações de longo prazo, bem como a reabilitação da pessoa que usa SPA. Essa pesquisa, revelou que isso somente é possível, a partir da utilização de ferramentas que ajudem na incorporação dos familiares na assistência em saúde mental. A inclusão pode ocorrer a partir da criação de ambientes apropriados para o diálogo e fortalecimento do vínculo da família com a equipe de saúde e que ademais, propiciem negociações de cuidado importantes com usuários e familiares (Oliveira et al., 2016).

O processo atrelado as decisões relacionadas a internação para os usuários enquanto uma opção terapêutica, revela-se enquanto barreira para o serviço de ordem comunitária, quando se trata das concepções dos familiares sobre o uso de drogas. A família com suas dificuldades de encarar o problema, encontra na internação, o procedimento adequado para resolver a sobrecarga de cuidados no lar (Silveira et al., 2016), contudo, cabe refletir sobre a eficácia desta terapêutica, quando não está em comum acordo com a pessoa em sofrimento.

A equipe do CAPS AD, quando integra a família no cuidado, deve trabalhar com preconceitos sociais, historicamente construídos e a inaptidão desta, em solucionar as questões relacionadas ao uso de drogas, pois, além de tudo, são questões que resultam em sofrimento familiar. Neste sentido, o cuidado a família deve ser pensado cotidianamente no processo de trabalho dos profissionais de saúde, arquitetando espaços e momentos de escuta, grupo de familiares, acolhida e até mesmo atendimento psicológico. Essas atitudes, os aproximam do serviço. Sem o apoio familiar, o caminho para obtenção dos resultados positivos demonstra-se ainda mais árduos, no que tange a reabilitação psicossocial (Oliveira et al., 2016). Não obstante, a família deve ser incluída no PTS (Camargo et al., 2019). 
Torna-se fundamental para a concretização dessa estratégia, que os profissionais de saúde estejam capacitados para realizar essa aproximação da família com o serviço de saúde, afinal, essa estratégia de cuidado, além de trazer resultados positivos no processo terapêutico do usuário, qualifica serviço, torna-o ainda mais resolutivo, produz novas abordagens, novas experiências para cuidar de outros diversificados casos (Arela et al., 2016; Pinho et al., 2017; Santos et al., 2018; TiradoOtálvaro, 2016).

\section{Conclusão}

A partir deste estudo foi possível refletir sobre as boas práticas em saúde mental no cuidado às pessoas em sofrimento psíquico por uso de drogas no contexto psicossocial. Não somente, identifica-se que mesmo com um aparato políticolegislativo solidificado no Brasil no que tange o cuidado contextualizado pela abordagem psicossocial, centrado na pessoa, ainda há trabalhadores, instituições de saúde mental e práticas que vislumbram métodos terapêuticos únicos e exclusivamente sob a mudança de comportamento abrupta - a abstinência enquanto imposição, sem considerar a autonomia da pessoa em sofrimento psíquico.

No entanto, a revisão narrativa concedeu alicerce para refletir a respeito de outras formas de cuidado possíveis, as "boas práticas", que geram resultados positivos por adaptarem-se aos contextos de vida do usuário de drogas, planejar e propor em conjunto com ele e sua família metas alcançáveis no contexto psicossocial. Entende-se com as novas abordagens em saúde mental - que a terapêutica deve considerar o usuário enquanto participante ativo do processo de cuidado, não mais deve ser posta unilateralmente pelos profissionais de saúde, já que remete a modelos tradicionais e conservadores em psiquiatria que são ineficazes para a maioria dos sujeitos. Considerando as boas práticas de cuidado em saúde mental, o primeiro passo terapêutico além de respeitar a história de vida do usuário e não o julgar pelo contexto de uso de drogas - é compreender o que a pessoa consegue fazer e o que está disposta a fazer durante o processo de reabilitação psicossocial.

A humanização nos espaços de saúde, considerando a clínica ampliada, território/contexto do sujeito, redução de danos, acessibilidade, acolhimento, articulação das redes de atenção psicossocial, autonomia e reinserção social, precisam extrapolar os muros das discussões acadêmicas para serem partilhadas ainda mais com o cotidiano dos serviços que prestam assistência a essas pessoas. Cuidar sem o olhar moralizador, compreender o sujeito e o espaço que a droga ocupa no "vazio" sentido pela pessoa em sofrimento, traz possibilidades terapêuticas mais benéficas para os sujeitos implicados no processo de cuidado no contexto psicossocial (pessoa usuária, família e profissionais da saúde).

Em relação as pesquisas sobre a temática das "boas práticas em saúde mental" no cuidado aos usuários de drogas, foi visto que, há uma produção consistente e discussões enriquecidas sobre propostas de rompimento com o modelo hegemônico de cuidado, em que valorizam os direitos humanos e o cuidado pautado nas singularidades da pessoa em sociedade. Novos desafios se colocam a respeito do tema, a partir da tradução do conhecimento científico e sua utilização, práxi, na vivência dos profissionais, para consolidar a importância dos serviços de base territorial.

\section{Referências}

Alexander, B. (1978). Rat park. Psychopharmacology.

Antonacci, M. H. (2016). Avaliação de boas práticas em saúde mental na atenção psicossocial. Tese de doutorado. Universidade de São Paulo. Ribeirão Preto, SP, Brasil.

Arela, D. S. S., Sales, I. M. M., Silva, F. M. D., \& Monteiro, C. F. S. (2016). Health network assisting users of alcohol, crack, and other drugs. Escola Anna Nery - Revista de Enfermagem, 20(2), 296-302.

Botega, N. J. (2012). Prática psiquiátrica no hospital geral: interconsulta e emergência. Artemed.

Brasil (2004). Portaria n 2.197, de 14 de outubro de 2004. Redefine e amplia a atenção integral para usuários de álcool e outras drogas, no âmbito do Sistema Único de Saúde - SUS. Diário Oficial da União - 15/10/2004. 
Research, Society and Development, v. 10, n. 7, e23610716419, 2021

(CC BY 4.0) | ISSN 2525-3409 | DOI: http://dx.doi.org/10.33448/rsd-v10i7.16419

Camargo, P. O., Oliveira, M. M., Tavares, D. H., Langmantel, K., \& Ramos, C. I. (2019). Práticas de redução de danos e consumo de crack. Revista Portuguesa de Saúde Mental, 22, 35-40.

Pinho, L. B., Siniak, D. S., Silva, A. B., Araújo, L. B., \& Folador, B. (2017). Funcionamento de um centro de atenção psicossocial para o atendimento a usuários de crack. Cuidado é fundamental, 9, 1099-06.

Franchini, B., Rodrigues, C. G. S. S., \& Tavares, D. H. (2017). Para além da droga: contribuições do centro regional de referência. Santa Cruz, 4a ed.

Hart, C. (2013). Um preço muito alto. Jorge Zahar.

Oliveira, G. C et al. (2016). A reabilitação psicossocial: processo de reconstrução da subjetividade do usuário de drogas. Revista Enfermagem UERJ, 23(6), $811-816$.

Santos, J. M., Baptista, J., Nasi, C., \& Camatta, M. W. (2018). Responsabilização e participação: como superar o caráter tutelar no centro de atenção psicossocial álcool drogas? Revista Gaúcha de Enfermagem, 39, 1-9.

Silva, A., Pinho, L. B., Oslchowsky, A., Kantorski, L. P., \& Pires, C. Z. (2018). Território: conceitos de profissionais de saúde mental no cuidado ao usuário de crack. Revista Presencia Salud Mental, Investigacion y Humanidades, 14, 11616-30.

Silva, S. N., Lima, M. G., \& Ruas, C. M. (2018). Avaliação de serviços de saúde mental brasileiros: satisfação dos usuários e fatores associados. Ciência \& Saúde Coletiva, 23(11), 3799-3810.

Silva, A. B., Pinho, L. B., Olschowsky, A., Siniak, D. S., \& Nunes, C. K. (2016). O cuidado ao usuário de crack: estratégias e práticas de trabalho no território. Rev Gaúcha Enferm, 38, 1-7.

Silva, M. N. R. M. O., Abbad, G. S., \& Montezano, L. (2018). Dinâmica organizacional e o modelo psicossocial de três centros de atenção psicossocial álcool e drogas. pesquisas e práticas psicossociais, 13(2), 1-17.

Silveira, E. A. A., Oliveira, P. P., Correio, P. M., Santos, W. J \& Rodrigues, A. B. O. (2016). Cuidado aos dependentes químicos: com a palavra profissionais de saúde de centros de atenção psicossocial em álcool e drogas. Cuidado é Fundamental, 8(2), 4347-4364.

Tavares, D. H., Silva, A. B., Pavani, F. P., Nunes, C. K., Franchini, B \& Rodrigues, C. G. S. S. (2021). Práticas de cuidado em um CAPS-AD: sobre a abstinência e redução de danos. Research, Society and Development, 10(6), 1-12.

Tirado-otálvaro, A. F. (2016). El consumo de drogas en el debate de la salud pública (2016). Cadernos de Saúde Pública, 32(7), 1-11.

Thornicroft, G \& Tansella, M. (2010). Boas práticas em saúde mental comunitária. Manole.

Zillmer, J. G. V \& Díaz-Medina, B. A. (2018). Revisión narrativa: elementos que la constituyen y sus potencialidades. J. Nurs. Health, 8(1), 1-2. 